\title{
SISTEMA DE TRATAMIENTO DE AGUAS RESIDUALES DEL MATADERO MUNICIPAL DE TACNA
}

\section{PROPOSAL FOR THE IMPROVEMENT OF THE WASTEWATER TREATMENT SYSTEM OF THE MUNICIPAL SLAUGHTERHOUSE OF TACNA}

\author{
YANNELA DaYanNa HuARACHI NúÑEZ ${ }^{1}$ \\ (D) https://orcid.org/0000-0003-3240-2533 \\ Cesar huanacuni lupaca ${ }^{2}$ \\ (D) https://orcid.org/0000-0002-1148-4050
}

Información del artículo: Recibido: 21/01/2021

Aceptado: 01/06/2021

Publicado: 28/06/2021

${ }^{1}$ Escuela de Ingeniería Ambiental, Universidad Privada de Tacna ${ }^{2}$ Docente en la Escuela de Ingeniería Ambiental, Universidad Privada de Tacna E-mail: ${ }^{1}$ yannelahn@outlook.es, ${ }^{2}$ chuanacuni@yahoo.es 


\section{Resumen}

Se tuvo como objetivo dar una propuesta de mejora al sistema de tratamiento de aguas residuales provenientes del proceso de beneficio animal del Matadero Municipal de Tacna, "Mario Reynaldo Eyzaguirre Yañez", con el fin de reducir los impactos generados por esta actividad. El diagnóstico situacional del área se realizó por medio de una lista de chequeo y control aplicado al sistema de tratamiento de aguas residuales, acorde a lo estipulado por el Reglamento Nacional de Edificaciones (RNE) OS. 0.90 Planta de Tratamiento de Aguas Residuales(PTAR), la cual mostró la ausencia de manuales de operación y mantenimiento, estructuras de rebose, cerco perimétrico, cribas, desarenador, medidor de caudal y sedimentador secundario. La caracterización de los afluentes y efluentes del tratamiento de aguas residuales se hizo mediante la toma de muestras en 4 puntos de la PTAR, determinando que, únicamente el parámetro de Grasas y Aceites cumple con lo estipulado; a su vez, para la medición de caudal se hizo uso de la metodología propuesta por el Ministerio de Agricultura y Riego, dando como resultado un caudal promedio de $0.82 \mathrm{l} / \mathrm{s}$, caudal mínimo $0.45 \mathrm{l} / \mathrm{s}$ y $1.99 \mathrm{l} / \mathrm{s}$ de caudal máximo. Con el fin de elegir un tratamiento adecuado, se determinó los reactores anaerobios de flujo ascendente como el tratamiento adecuado para este tipo de aguas residuales, siendo este, acompañado por las unidades necesarias para un buen desempeño, tales como: cribas, sedimentador primario, trampa de grasas, sedimentador secundario y nitrificación-desnitrificación. Finalmente se propone una serie de alternativas para la reducción de residuos al tratamiento de aguas residuales del beneficio animal.

Palabras claves: Aguas Residuales, Matadero, Beneficio animal, Valores máximos admisibles, Manejo de residuos líquidos.

\section{Abstract}

The objective was to propose an improvement to the wastewater treatment system of the animal processing plant of the Municipal Slaughterhouse of Tacna, "Mario Reynaldo Eyzaguirre Yañez", in order to reduce the impacts generated by this activity. The situational diagnosis of the area was carried out by means of a checklist and control applied to the wastewater treatment system, in accordance with the National Building Regulations (RNE) OS. 0.90 Wastewater Treatment Plant (WWTP), which showed the absence of operation and maintenance manuals, overflow structures, perimeter fencing, screens, desander, flow meter and secondary sedimenter. The characterisation of the influents and effluents of the wastewater treatment was done by taking samples at 4 points of the WWTP, determining that only the parameter of Fats and Oils complies with the stipulated; at the same time, for the flow measurement, the methodology proposed by the Ministry of Agriculture and Irrigation was used, resulting in an average flow of $0.82 \mathrm{l} / \mathrm{s}$, minimum flow $0.45 \mathrm{l} / \mathrm{s}$ and $1.99 \mathrm{l} / \mathrm{s}$ of maximum flow. In order to choose a suitable treatment, upflow anaerobic reactors were determined as the appropriate treatment for this type of wastewater, accompanied by the units necessary for good performance, such as: screens, primary sedimentation, grease trap, secondary sedimentation and nitrification-denitrification. Finally, a series of alternatives for waste reduction are proposed for the treatment of animal processing wastewater.

Keywords: Wastewater, Slaughterhouse, Animal processing, Maximum admissible values, Liquid waste management. 


\section{Introducción}

Uno de los problemas ambientales que ha cobrado importancia a nivel mundial son los volúmenes elevados de aguas residuales sin tratamiento, tanto urbanas como industriales, relacionado a este factor, se debe considerar el acelerado crecimiento poblacional en regiones de Costa y Sierra, donde, por ende, se desarrollan mayoresactividades industriales. La Región de Tacna, en Perú, cuenta con una población de 296788 (Instituto Nacional de Estadística e Informática, 2018), donde diariamente existe una gran demanda de productos cárnicos, es por eso, que el Matadero Municipal de Tacna incrementa el beneficio de animales destinados al abasto público, dando lugar a un aumento de la generación de residuos sólidos y líquidos los cuales son vertidos sin adecuado tratamiento, siendo esto una fuente de contaminación ambiental. El Matadero Municipal de Tacna Mario Reynaldo Eyzaguirre Yañez, produce residuoslíquidos tales como sangre, aguas de lavado, orines de animales, entre otros, los cuales son vertidos en el alcantarillado público luego de ser tratada por un sistema que consta de un sedimentador, percolador y un pozo séptico, los cuales, según informe emitido por la Empresa Prestadora de Servicios (EPS) no cumplen con la remoción suficiente para dar cumplimiento del D.S. N¹0-2019- VIVIENDA- Decreto Supremo que aprueba el Reglamento de Valores Máximos Admisibles (VMA) para las descargas de aguas residuales no domésticas en el sistema de alcantarillado sanitario, dando lugar pagos por exceso de concentración por parte de dicha empresa .

Considerando la falta de procesos en la planta de tratamiento de aguas residuales se presenta problemas tales como: colmatación de unidades (sedimentador, percolador y pozo séptico), foco de proliferación de vectores y malos olores, generando en conjunto malestar a los usuarios, trabajadores, vecinos y visitantes. Las actividades económicas tienden a deteriorar la calidad de suelo, aire y agua en diferente medida, una de las actividades a considerar en este punto son lasde beneficio animal, la cual, a la vez de generar problemas ambientales, produce problemas sociales y económicos siendo por tales motivos necesario de evaluación. Llevando esta problemática a nivel local, se identifica al matadero municipal de Tacna "Mario Reynaldo Eyzaguirre Yañez" como único matadero con autorizacióndel Servicio Nacional de Sanidad y Calidad Agroalimentaria (SENASA) para brindar el servicio de beneficio a bovinos, porcino, ovinos y caprinos en la provincia de Tacna. Considerando las actas de supervisión ambiental emitidas por las entidades de Fiscalización Ambiental, tales como: Unidad de Gestión de Conservación y Fiscalización Ambiental de la Municipalidad Provincial de Tacna y la Unidad de Fiscalización y Control Municipal de Municipalidad Distrital de Pocollay, las cuales constataron en forma reiterativa el mal manejo de las aguas residuales del proceso de beneficio animal.

Los PTAR (puntos de tratamiento de aguas residuales) provenientes del beneficio animal, consta de un sedimentador, un pozo percolador y un pozo séptico, los cuales luego de 10 años desde su construcción, muestran problemas de mantenimiento y operación, lo cual no permite una correcta remoción de contaminantes trayendo esto como problema principal el no cumplimiento con los VMA establecidos por D.S. N¹0-2019-VIVIENDA y como problemas secundarios a la proliferación de vectores y emanaciónde malos olores. Teniendo en cuenta que la PTAR del matadero municipal de Tacna tiene como unidades un sedimentador, un pozo séptico y un percolador, se debe saber que, por errores de diseño, poco mantenimiento o mal uso del tratamiento, el proceso inadecuado, generaría que las unidades del tratamiento colapsen y/o no remueva los contaminantes de la forma esperada.

El presente trabajo, da a conocer las condiciones actuales de funcionamiento del tratamiento de aguas residuales provenientes del proceso de beneficio animal, debido a que es inapropiado dar conjeturas de lafuncionabilidad del tratamiento al no haberse realizado una investigación previa. La implementación de mejoras en el tratamiento de las aguas residuales por parte de la administración del matadero municipal ayudaría a reducir -en comparación a la multa previamente impartida por la Empresa Prestadora de Servicio Tacna - e incluso eliminar futuros pagos por vertimiento con exceso de concentraciones al servicio de alcantarillado público, a su vez, al dar un correcto tratamiento a las aguas residuales, se dará cumplimientos a los compromisos acordados con el OEFA y la Municipalidad 
Distrital de Pocollay, evitando así la clausura temporal o definitiva del matadero por parte de estas entidades lo cual generaría un déficit en los gastos propios, causando daños económicos a la MunicipalidadProvincial de Tacna al no tener los ingresos usuales, los cuales cubren costospor pagos a personal que labora en planta.

Según Morán (2014) la implementación de una PTAR da como resultados la disminución de diversos parámetros por medio de percoladores, tales como: $\mathrm{DBO}_{5}$ de $216,0 \mathrm{mg} / \mathrm{l}$ a $24,58 \mathrm{mg} / \mathrm{l}$, grasas y aceites de $22,3 \mathrm{mg} / \mathrm{la} 0 \mathrm{mg} / \mathrm{l}$. Según Benavides (2006) al evaluar la planta de tratamiento menciona que existe una remoción de un $33,31 \%$ de $\mathrm{DQO}, 15,00 \%$ de $\mathrm{DBO}_{5}$ y $11,46 \%$ de grasas y aceites, por medio de lodos activados como tratamiento secundario. Según Quille \& Donaire (2013) el tratamiento de aguas residuales provenientes del beneficio animal por medio de tratamiento mixto de CalFloculación, da resultados positivos, con una remoción de un $75 \%$ deDBO ${ }_{5}$, un $73 \%$ de $\mathrm{DQO}$ y un $99 \%$ de reducción de los sólidos suspendidos totales. Así mismo, según Espinoza (2017), dichas aguas pueden ser tratadas hasta el punto de poder ser reutilizadas para riego de plantas de tallo corto mediante el uso de tratamiento biológico, ha mostrado resultados positivos, tales como la remoción de $\mathrm{DBO}_{5}$ de 2209,33 a 7,36 mg/L,DQO de 2602,66 a $24,77 \mathrm{mg} / \mathrm{L}$, SST 44,5 a 0,36 mg/L/h, Grasas y Aceites de37,7 a 0,82 mg/L. Finalmente, según Rubio \& Padilla (2009), con el uso del sistema biológico ya nombrado, se logra la remoción de un $53,9 \%$ de materia orgánica y $48,72 \%$ de DQO.

La Municipalidad Provincial de Tacna por medio de unidad de Gestión de Conservación y Fiscalización emitió informes los cuales manifiestan la necesidad de implementar una planta de tratamiento de aguas residuales luego de la supervisión dada a dicho local, se informa a su vez el mal estadodel tratamiento y su mala disposición final de los lodos generados, siendo estoreiterado en actas de supervisión ambiental emitidas por la misma institución. A su vez, en Actas de verificación sanitaria a Mataderos emitidas por el Servicio Nacional de Sanidad Agraria (SENASA), se pone en conocimiento la presencia de vectores alrededor del matadero, siendo esta observación dada de forma reiterativa. Finalmente, la Municipalidad de Pocollay por medio de la Unidad de Fiscalización y Control Municipal, corrobora en Actas de Constatación Municipal la presencia de vectores, canaletas descubiertas y residuos sólidosen el tratamiento de aguas residuales.

Mataderos Municipales: Son las instalaciones de procesamiento de carne administradas por autoridades locales, las cuales tienen como propósito brindar a la población productos cárnicos que cumplan con las normativas de sanidad y calidad parael consumo humano seguro. (Taveras, María A, et al, 2011)

Aguas residuales de mataderos municipales: Las aguas residuales de las plantas de beneficio animal, se caracterizan por tener una composición compleja, ya que, tanto el volumen como las concentraciones de los contaminantes, son mucho mayores a los efluentes domésticos comunes (Carrasquero, 2015) (Bustillo-Lecompte, 2015).

En los efluentes se de este tipo de industria se suele encontrar: sangre, rumen, pelos, grasas y proteínas, lo que conlleva a elevadas concentracionesde materia orgánica, coliformes totales, sólidos suspendidos, grasas, nitrógeno y fósforo, esto ocasionado por las actividades de beneficio, lavado de carcazas y limpieza de equipos e instalaciones (Gilberto Salas C., 2008) (Cammarota, 2006), y si bien estos valores son altos, son adecuados por tener un contenido de compuestos orgánicos altos lo que aumenta el índice de biodegrabilidad (Mittal, 2006).

Según informes presentado por la Unidad de Gestión de Conservacióny Fiscalización Ambiental de la Municipalidad Provincial de Tacna, la Unidad de Fiscalización y Control Municipal de la Municipalidad Distrital de Pocollay y actas de verificación sanitaria a mataderos realizadas por SENASA, se recomienda a la administración del Matadero Municipal de Tacna implementar una PTAR adecuada para las aguas generadas en la actividad de beneficio ya que se evidenció la presencia de vectores y malos olores, dicha implementación, reduciría los impactos ambientales generados los cuales traen consigo afectaciones a la salud pública. Por los motivos ya señalados, es que nace la necesidad de dar una propuestade mejora al tratamiento de aguas residuales, logrando con esto, la reducción de las concentraciones de los parámetros en el vertimiento al alcantarillado y la disminución de vectores, 
siendo, en definitiva, el medio ambiente y la sociedad en general, los beneficiarios de estas mejoras.

\section{Objetivo}

Elaborar una propuesta de mejora del sistema de tratamiento de aguas residuales del matadero municipal de Tacna.

\section{Metodología}

El presente informe de tesis es de tipo exploratorio y diseño de campo. EL Matadero Municipal de Tacna, se encuentra ubicado en el sector Vilauta, distrito de Pocollay, provincia de Tacna, región Tacna, República de Perú (Municipalidad Provincial de Tacna, 2015). Cuenta con un área total de, 21504.99 metros cuadrados $\left(\mathrm{m}^{2}\right)$, donde anualmente, en promedio, se sacrifican 9600 cabezas de ganado bovino, 39500 de porcino, 350 de ovino y 200 de caprinos, según estadística de beneficio del año 2019.

Con respecto a la planta de tratamiento de aguas residuales industriales, esta consta de 3 unidades: un sedimentador, un percolador, un pozo séptico, una caja derivadora y 2 unidades que cumplen la función de almacenamiento (Municipalidad Provincial de Tacna, 2015). Para dar cumplimiento al objetivo general, se consideró necesario la realización de diferentes actividades, las cuales responden a los objetivos específicos planteados. Es por tal motivo que se consideró necesaria la realización de un diagnóstico del área en estudio el cual constó de los siguientes pasos:

- Identificación de las unidades que componen PTAR de las aguas del proceso de beneficio mediante la observación de los mismos.

- Aplicación de una lista de chequeo a la PTAR, a fin de identificar la presencia o ausencia de diversos puntos estipulados por el Reglamento Nacional de Edificaciones (RNE) OS. 090 Plantas de Tratamiento de Aguas Residuales.

- Medición del caudal del agua residual cruda (afluente) de la PTAR por el método del flotador, realizando el procedimiento del Manual $\mathrm{N}^{\circ} 5$ Medición de agua del Ministerio de Agricultura y Riego del año 2015.

\section{Tabla 1}

Determinación del factor de corrección Fc para el cálculo de caudales por el métododel flotador

La evaluación de calidad de agua tratada, la misma que se realizó según lo estipulado por D.S. 010-2019- VIVIENDA en el capítulo IV artículo 12 referido a las tomas de muestras, Los puntos establecidos para la toma de muestra fueron los siguientes: Aguas crudas (Punto 1), salida del sedimentador (Punto2), salida del Percolador (Punto 3) y Salida del tratamiento (Punto 4) figura 1: 


\section{Figura 1}

Diagrama del sistema de tratamiento de aguas residuales del proceso de beneficio del Matadero Municipal de Tacna

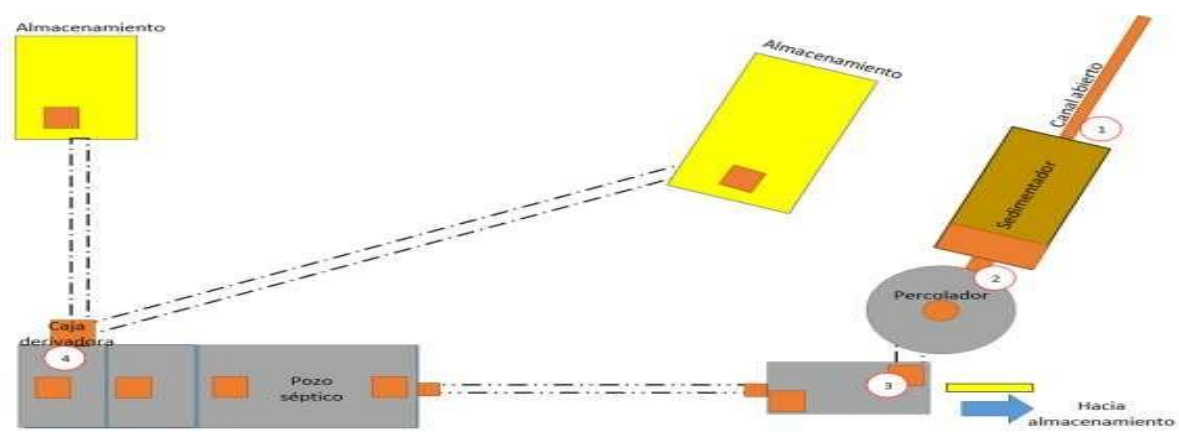

Las muestras tomadas fueron enviadas al laboratorio ALS LS Perú S.A.C, lugardonde se hizo la evaluación de los siguientes parámetros: DBO, DQO, G y A, Sólidos Disueltos y Nitrógeno amoniacal, siendo estos parámetros comparados con los VMA estipulados en el D.S N 010-2019 - VIVIENDA Decreto Supremoque aprueba el reglamento de Valores Máximos Admisibles (VMA) para las descargas de aguas residuales no domésticas en el alcantarillado sanitario y R.M. 360-2016-VIVIENDA

Uso del método de factores ponderados para la elección del tratamiento de aguas residuales, el cual comenzó con la identificación de factores relevantes para la toma de decisiones, seguido de la ponderación entre ellos según su importancia relativa, culminando con la puntuación de la alternativa para cadauno de estos criterios bajo la escala determinada, obteniéndose con esto una calificación global de las alternativas.

Finalmente, en bases a las observaciones en el área de beneficio animal, se dio alternativas para la reducción de residuos que pudieran influir en la planta de tratamiento de aguas residuales.

Para el análisis de los datos se utilizará el análisis descriptivo como criterio para la recopilación de información. La información hallada será procesada por programas tales como Excel parala generación de tablas, figuras, dando esto como resultado una base de datos. Con la información obtenida se elaborará las recomendaciones o propuestas planteadas para la optimización del tratamiento de aguas residuales del matadero municipal de Tacna.

\section{Resultados}

La PTAR del matadero municipal de Tacna "Mario Reynaldo Eyzaguirre Yañez" se encuentra ubicado dentro de las instalaciones del mismo, a unadistancia aproximada de 25 metros de la zona de beneficio y a aproximadade 30 metros de las viviendas aledañas.

Las aguas residuales del proceso de beneficio son direccionadas hacia laPTAR por medio de canaletas de $30 \mathrm{~cm}$. de ancho cubiertas con rejillas en platina de $3 / 4$ (Dentro de la zona de beneficio), las cuales conducen el agua a un canal de concreto de $30 \mathrm{~cm}$ de ancho, $30 \mathrm{~cm}$ de profundidad y 10 de grosor (Fuera de la zona de beneficio); el canal recorre un aproximado de 15 metros no lineales desde la salida del área de beneficiohasta el ingreso al sedimentador.

La PTAR es compuesta por un sedimentador primario de dimensiones: $5 \mathrm{~m}$. de largo, $1.5 \mathrm{~m}$. de ancho y $1 \mathrm{~m}$. de profundidad, con una capacidad noespecificada en documentos proporcionados por la administración del matadero municipal; un pozo percolador de $3,00 \mathrm{~m}$ de diámetro y 4,42 de profundidad; seguido de un pozo séptico de dimensiones: $9 \mathrm{~m}$. de largo y 3,20 m. de ancho, con una profundidad aproximada de $2 \mathrm{~m}$. 
El retiro del agua residual tratada es por medio de una cisterna, cuyo contenido es vertido en el último buzón de la troncal que se encuentra bajo la Av. Collpa.

La aplicación de una lista de chequeo, adaptada del Reglamento Nacional de Edificaciones OS. 090 Plantas de Tratamiento de Aguas Residuales. La aplicación de la lista de chequeo evidenció el cumplimiento del $36.4 \%$ delos Ítems estipulados en el Reglamento Nacional de Edificaciones OS. 090 Plantas de Tratamiento de Aguas Residuales.

Se realizó la medición de caudales afluentes de la PTAR por una semana, dela información recolectadas podemos determinar que el caudal promedio que ingresaa la PTAR. Según los datos hallados, podemos determinar que el día con mayor caudal afluente registrado y con más alto caudal promedio es el día viernes, con un valor máximo de $1.9 \mathrm{l} / \mathrm{s}$, el día con menor caudal registrado, diferente a cero, es el día lunes con $0.45 \mathrm{l} / \mathrm{s}$.

Los caudales varían usualmente entre $0.5 \mathrm{l} / \mathrm{s}$ y $1.0 \mathrm{l} / \mathrm{s}$, sin embargo, existe una varianza entre las 10 y las 11 horas con un caudal superior al mencionado, se presume que se trata de ingreso de agua a causa de la actividad de lavado de vísceras y/o vertido de agua de escaldado. Se afirmar que el comportamiento de loscaudales horarios es muy errático.

Las siguientes figuras muestran la variación de concentraciones de los parámetros en estudio; los puntos de muestreo AR-1, AR-2, AR-3 y AR-4 corresponden a las aguas residuales crudas, salida del sedimentador primario, salidade percolador y salida de pozo séptico respectivamente.

\section{Figura 2}

Variación de la concentración de la Demanda Bioquímica de Oxígeno(DBO5)(mg/l) en el sistema de tratamiento de aguas residuales

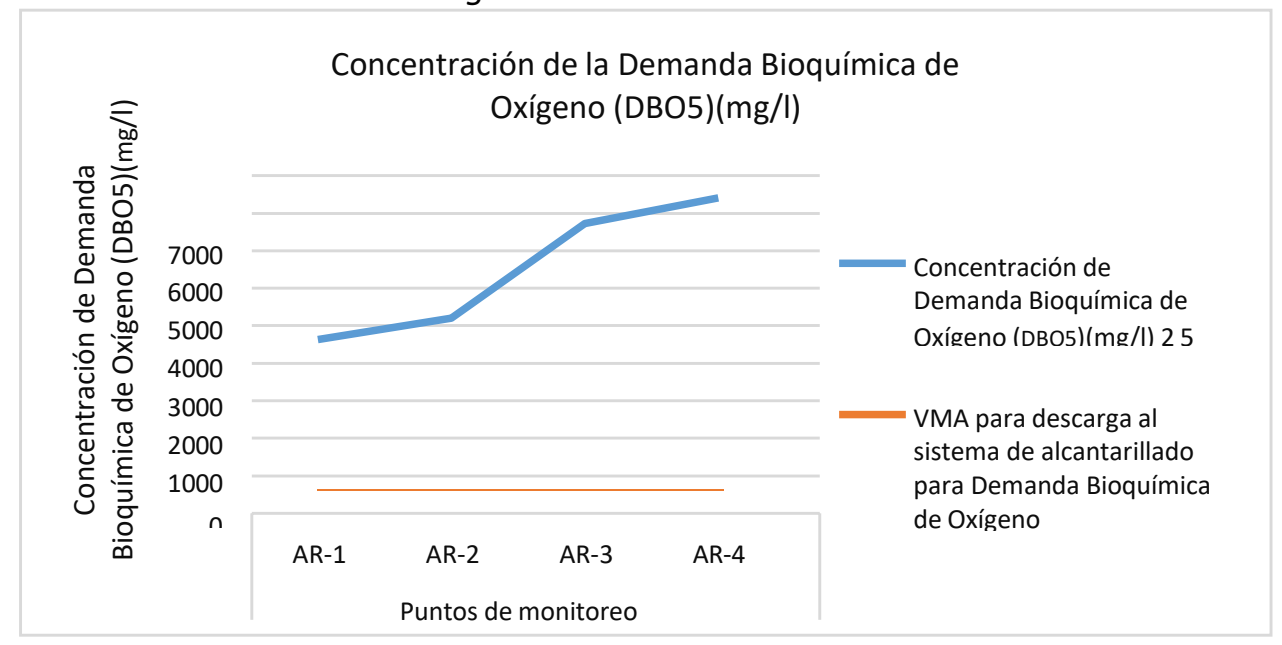

Observamos el incremento progresivo de la concentración de la Demanda Bioquímica de Oxígeno, superando, de comienzo a final del tratamiento, los ValoresMáximos Admisibles. 


\section{Figura 3}

Variación de la concentración de Demanda Química de Oxígeno (DQO) (mg O2/L) en el sistema de tratamiento de aguas residuales

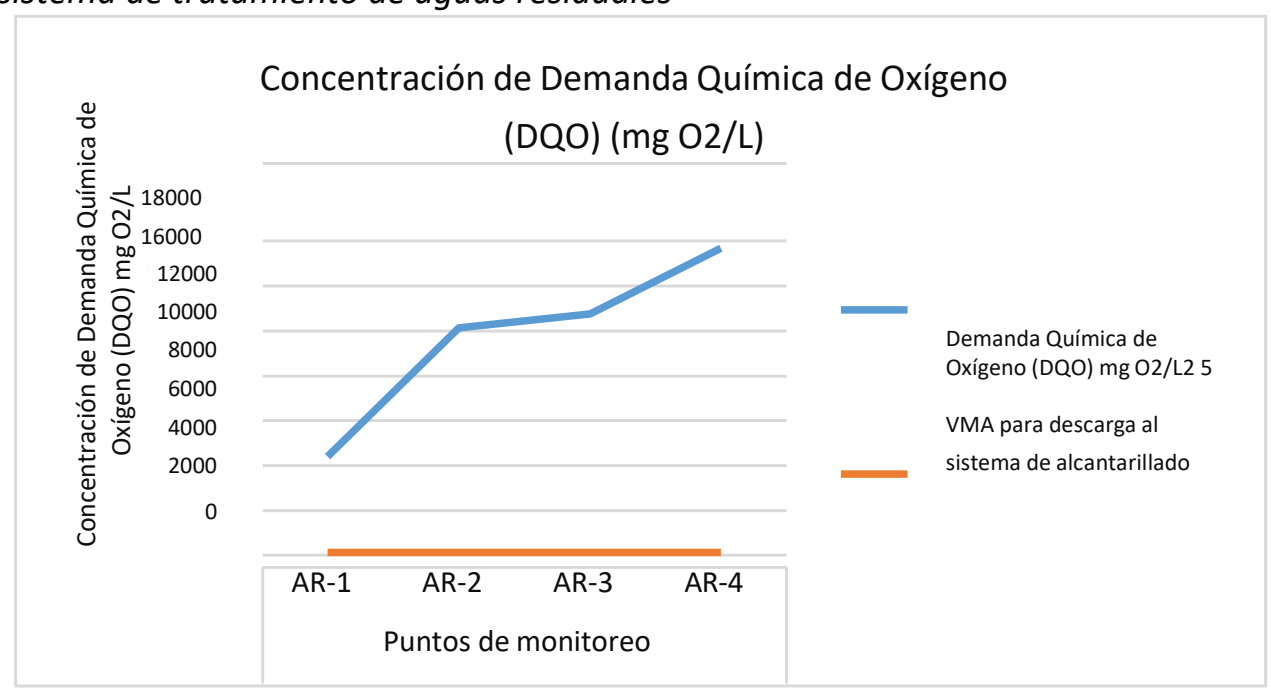

Observamos el incremento progresivo de la concentración de la Demanda Química de Oxígeno, superando, de comienzo a final del tratamiento, los Valores Máximos Admisibles.

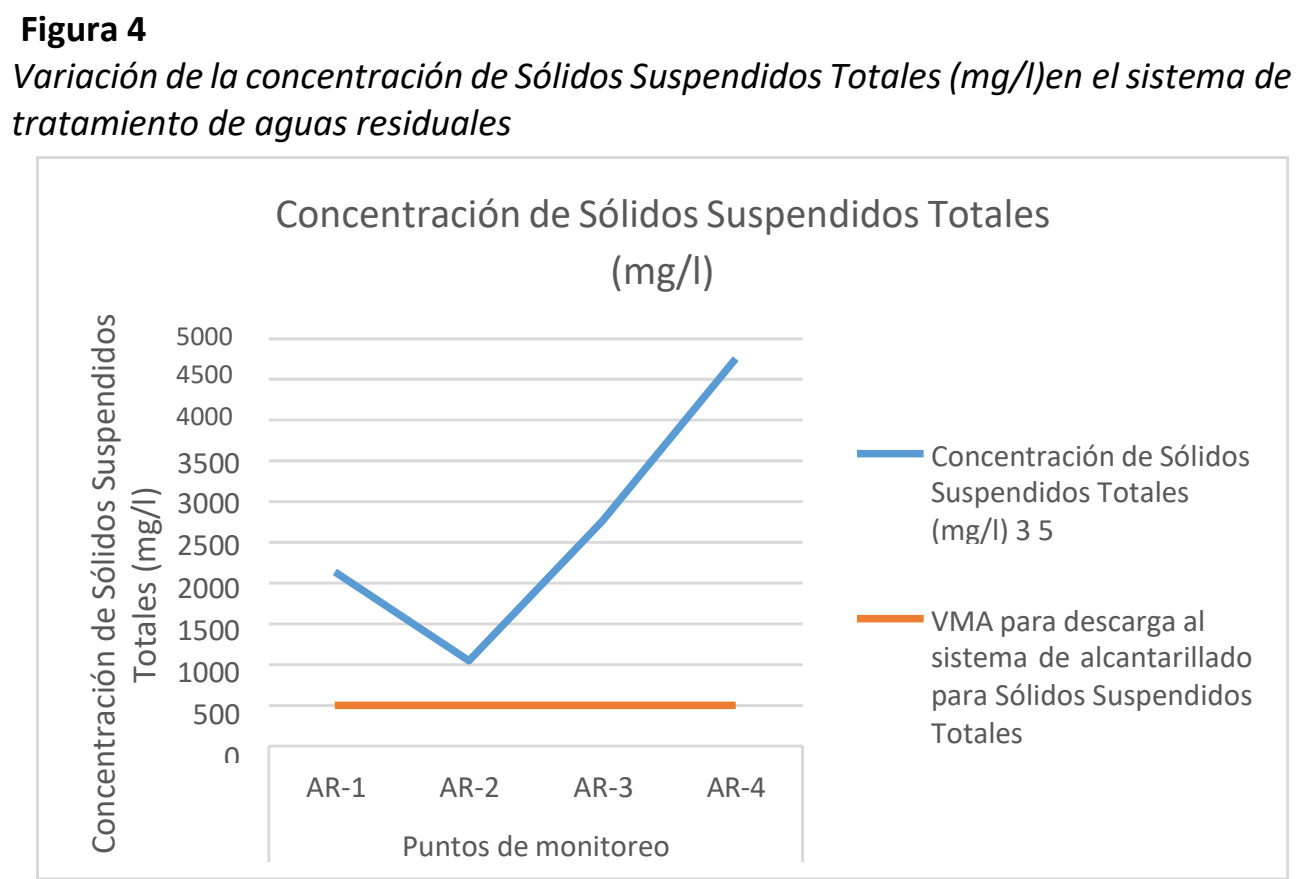

Observamos la disminución de concentración del parámetro al pasar por el sedimentador primario, seguido del aumento en los siguientes procesos, siendo la concentración, de comienzo a final del tratamiento, mayor a lo establecido los ValoresMáximos Admisibles. 


\section{Figura 5}

Variación de la concentración de Aceites y Grasas (mg/l en el sistemade tratamiento de aguas residuales

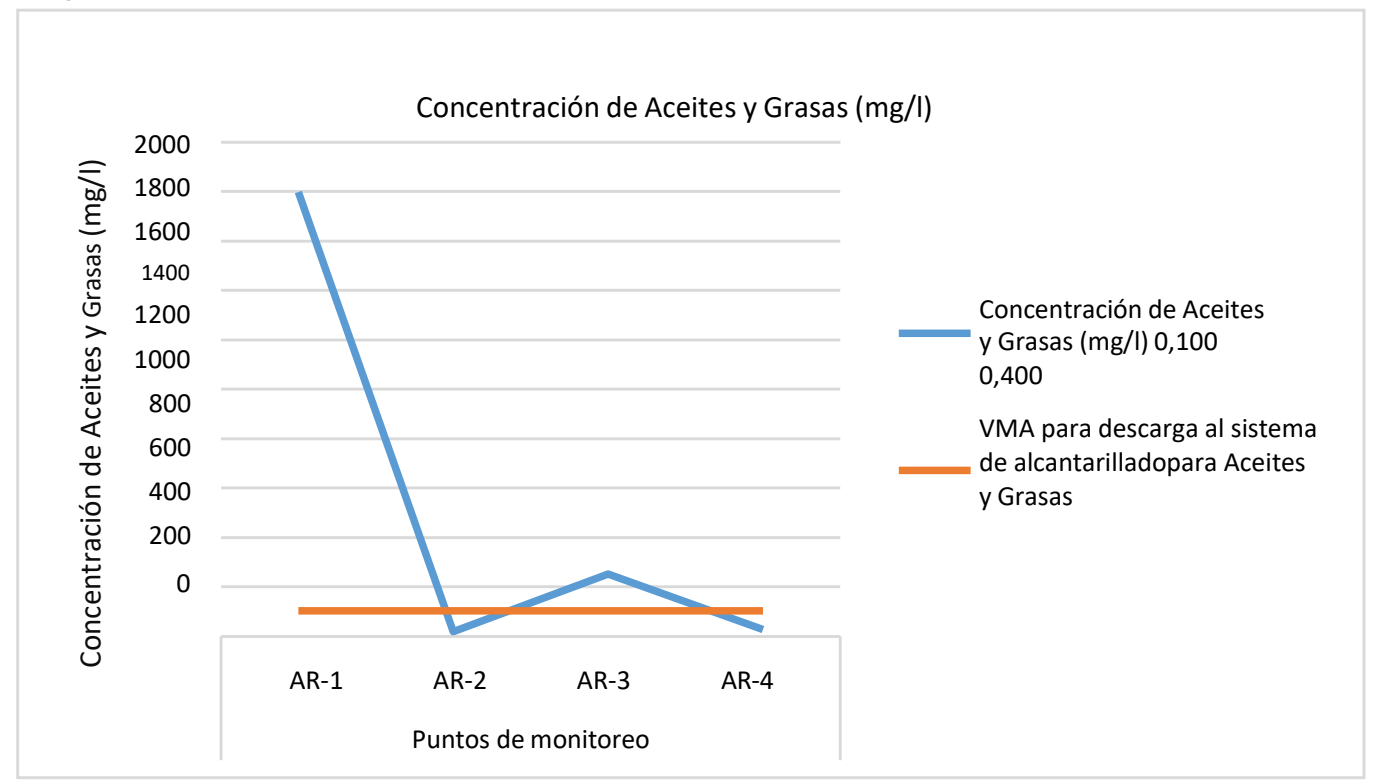

Se observó la disminución y aumento intercalado de la concentración de grasas y aceites al pasar por los diferentes procesos de la PTAR, presentando el agua residual al final del tratamiento una concentración menor a lo establecido por los Valores Máximos Admisibles.

\section{Figura 6}

Variación de la concentración de Nitrógeno Amoniacal, Amoniaco(mg NH3-N/L)

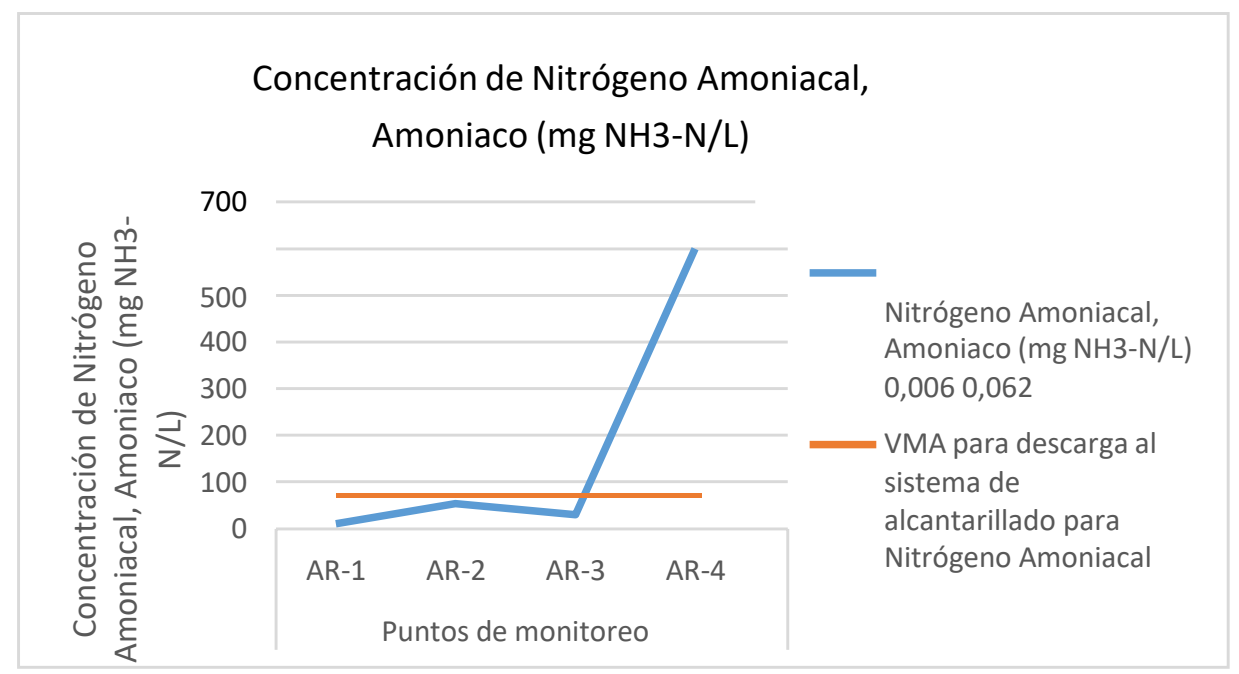

Se observó el aumento y disminución intercalado de la concentración denitrógeno amoniacal al pasar por los procesos de la PTAR, conteniendo el agua residual al final del tratamiento una concentración mayor a lo establecido por losValores Máximos Admisibles

De la evaluación de los resultados emitido por Informe $N^{\circ} 514 / 2020$ e InformeNำ15/2020, los parámetros establecidos en el D.S.N010-2019-VIVIENDA talescomo: Demanda Bioquímica de Oxígeno, Demanda Química de Oxígeno, Sólidos Suspendidos Totales y Nitrógeno Amoniacal tienen una 
concentración superior alo especificado por la norma teniendo como causa probable la falta de unidades de pre y post tratamiento a su vez de la falta de dimensionamiento de las unidadesen base a una caracterizaciones de las aguas provenientes del beneficio animal.

\section{Evaluación y Selección del tratamiento para los efluentes}

Se consideró las ventajas y desventajas del tratamiento por escoger, por tal motivo se determinó los siguientes factores:

a) Eficiencia de remoción: Disminución de concentración de DBO, DQO y SST

b) Costos de operación y mantenimiento: Dentro de los parámetros a considerar en la elección de un tratamiento es la evaluación de costos de mano de obra, costos de energía, herramientas y materiales.

c) Área de trabajo: Área requerida para la instalación de un tratamiento de aguas residualeslo cual debe ser menor al área disponible en el matadero municipal.

d) Consumo de energía: Factor importante en la toma de decisión en consideración del reducido presupuesto designado al tratamiento de aguas residuales.

e) Mano de Obra: El mantenimiento de tratamientos de aguas requiere de la presencia de personal calificado para la materia, lo cual elevaría el presupuesto designado.

f) Generación de lodos: Este factor determina la cantidad de residuos sólidos generados por el tratamiento, necesario la consideración de este factor al poder necesitar un presupuesto para su disposición final o posible reutilización, siendo la cantidad de residuos sólidos y presupuesto directamente proporcional.

La confrontación de factores se realizó aplicando el criterio de relación, siendo esto determinado por medio de relación y conexión, dando el valor de 1 en caso hubiera relación entre factores y el valor 0 en caso de no existir relación.

Tabla 2

Análisis entre factores ponderados

\begin{tabular}{|c|c|c|c|c|c|c|c|c|}
\hline Factores & A & B & $C$ & D & $E$ & $F$ & Conteo & $\begin{array}{l}\text { Ponderado } \\
\text { (\%) }\end{array}$ \\
\hline A & - & 1 & 1 & 1 & 1 & 1 & 5 & 22,73 \\
\hline B & 1 & - & 1 & 1 & 1 & 1 & 5 & 22,73 \\
\hline C & 1 & 1 & - & 0 & 1 & 1 & 4 & 18,18 \\
\hline D & 1 & 1 & 0 & - & 0 & 0 & 2 & 9,09 \\
\hline$E$ & 1 & 1 & 1 & 0 & - & 0 & 3 & 13,64 \\
\hline$F$ & 1 & 1 & 1 & 0 & 0 & - & 3 & 13,64 \\
\hline \multicolumn{7}{|c|}{ Total } & 22 & 100 \\
\hline
\end{tabular}

Tabla 3

Rango de calificación

\begin{tabular}{cc}
\hline Escala & Puntaje \\
\hline Excelente & 100 \\
Muy buena & 80 \\
Buena & 60 \\
Regular & 40 \\
Mala & 20 \\
\hline
\end{tabular}


Considerando la elección de Reactores anaerobios de flujo ascendente como tratamiento secundario de las aguas residuales provenientes de la actividad de beneficio, se presenta el siguiente flujo con las características de dichos reactores.

\section{Figura 7}

Diagrama del Proceso de Tratamiento de Aguas Residuales Propuesto para las aguas residuales del proceso de beneficio del Matadero Municipal de Tacna

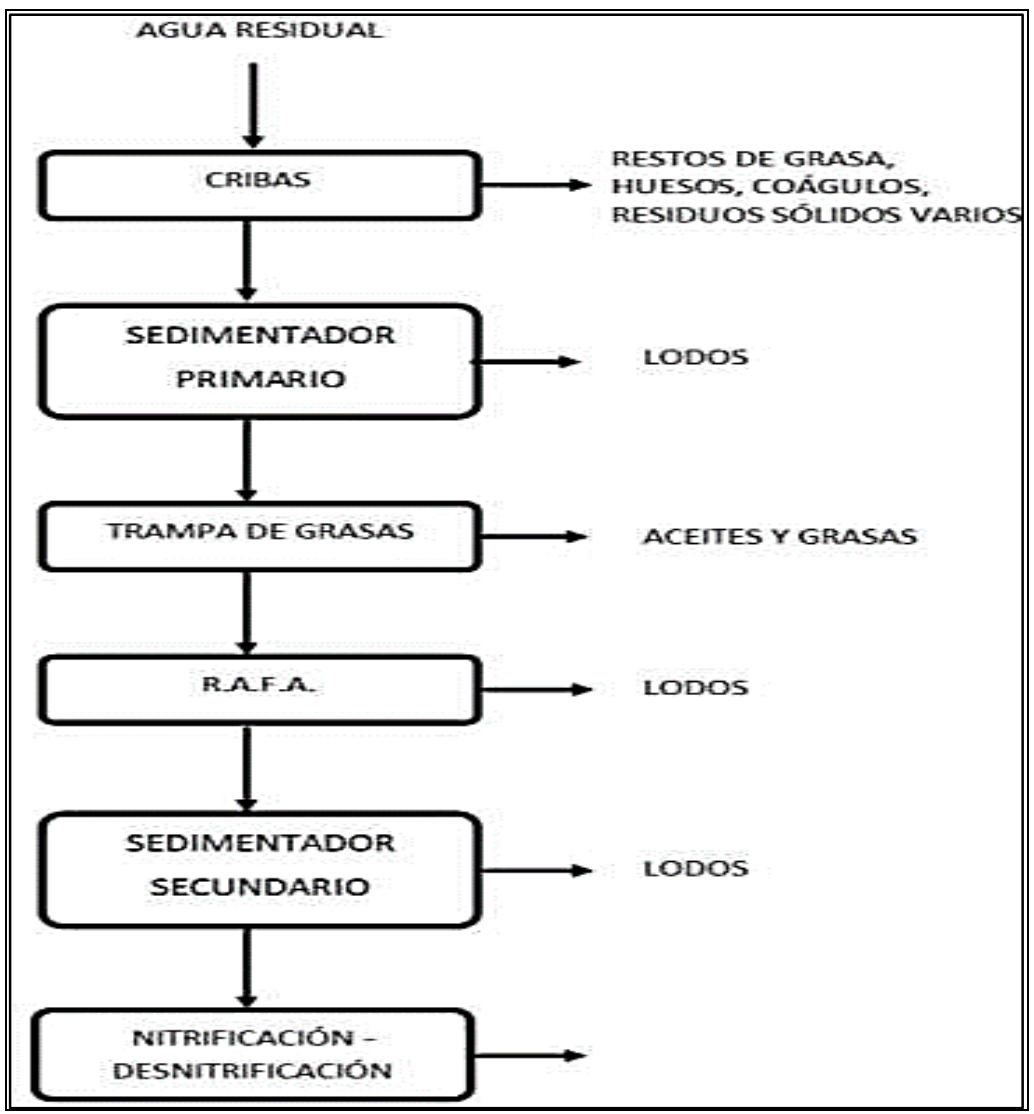

El tratamiento seleccionado como adecuado fueron los Reactores Anaerobios de Flujo Ascendente, al recibir mayor puntaje en comparación a los demás tratamientos.

En todo tratamiento de aguas residuales es de necesaria implementación el tratamiento preliminar, ayudando esto a la separación del agua residual de cualquiermaterial que pueda generar problemas tales como atoro de tuberías, entre otros. Es por tal motivo la necesaria inclusión de rejillas de desbaste de tipo manual o automático, lo cual ayudaría a la remoción de trozos de grasa, pelos, restos de huesos, coágulos, ente otros; como siguiente unidad necesaria al PTAR encontramosa la Trampa de grasas, lo cual permitiría la eliminación de grasas y aceites a su vezde material flotante de menor densidad; en consideración a la elección de tratamientosecundario a un reactor anaerobio de flujo ascendente, en base a la norma OS.090 y a los resultados de la caracterización de las aguas residuales, es necesario la inclusión de un sedimentador primario previo al tratamiento secundario y un sedimentador secundario post RAFA.

Teniendo en cuenta el exceso de concentración de Nitrógeno amoniacal para dar cumplimientos de los VMA, es que es necesaria la implementación de un tratamiento terciario tal como Nitrificación - Des nitrificación.

Considerando la elección de Reactores anaerobios de flujo ascendente como tratamiento 
secundario de las aguas residuales provenientes de la actividad de beneficio, se presenta el siguiente flujo con las características de dichos reactores.

\section{Discusión}

La aplicación de las propuestas de reducción en los factores que influyen en elfuncionamiento de la PTAR lograría una reducción considerable de la carga orgánica de las aguas residuales dando como resultado un mejor funcionamiento de la PTAR, menor de generación de olores, disminución en laacumulación de residuos sólidos en los procesos y reducción de presencia de vectores, siendo el principal beneficiario de dichos cambios el medio ambientea su vez de la población aledaña al área de estudio, la cual reduciría en gran medida su desaprobación a las actividades realizadas por el matadero.

Se encontraron concentraciones superiores en los parámetros de $\mathrm{DBO}_{5}, \mathrm{DQO}, \mathrm{SST}$ y Nitrógeno Amoniacal, los mismos que son establecidos por el D.S N010-2019-VIVIENDA, coincidiendo con Oficio N¹260-2019/300.700/EPSTACNA S.A. el cual notifica el exceso de concentración de los VMA., por tal motivo es que se determina que existe la colmatación y/o mal diseño de las actuales unidades de la PTAR.

La selección adecuada de alternativas de tratamiento de aguas residuales, es de vital importancia para la remoción correcta concentraciones de parámetros como DBO5, DQO, SST y Nitratos, los cual evitará el exceso de concentraciónde los VMA e incluso como lo indica Peralta (2017), el uso de las aguas residuales tratadas de un matadero municipal para el riego de plantas de tallo corto bajo los parámetros sugeridos por la Organización Mundial de la Salud.

El dar un valor agregado a los residuos generados en el proceso de beneficio,traería múltiples ventajas: el uso de la sangre para la elaboración de morcilla, harina de sangre, entre otros, disminuiría la carga orgánica de las aguas residuales, ya que la sangre no ingresaría a la PTAR junto con las aguas crudas. La valorización del contenido ruminal daría como resultado una menorpresencia de los mismos en la PTAR, evitando esto problemas usuales como la obstrucción de los canales y la colmatación de las unidades (sedimentador, percolador y pozo séptico).

\section{Conclusiones}

Se plasmó la propuesta de mejora al funcionamiento del sistema de tratamiento de las aguas residuales de la actividad de beneficio del Matadero Municipal de Tacna "Mario Reynaldo Eyzaguirre Yañez", el cual consistió en recomendación de unidades que deben ser incluidos en la PTAR, a su vez de recomendaciones para la valorización de los residuos generados en el beneficio animal. La caracterización de los afluentes y efluentes del tratamiento de aguas residuales del proceso de beneficio, dieron como resultado para el parámetro $\mathrm{DBO}_{5}$ una concentración en el afluente de $4630 \mathrm{mg} / \mathrm{l}$ y del efluente de $8417 \mathrm{mg} / \mathrm{l}$,en el parámetro DQO, $6400 \mathrm{mg} / \mathrm{l}$ y $15667 \mathrm{mg} / \mathrm{l}, \mathrm{SST} 2136 \mathrm{mg} / \mathrm{l}$ y $4753 \mathrm{mg} / \mathrm{l}$, Aceites y Grasas, $1797 \mathrm{mg} / \mathrm{l}$ y $26.20 \mathrm{mg} / \mathrm{l}$ y Nitrógeno amoniacal $9.825 \mathrm{mg} / \mathrm{l}$ y $600.5 \mathrm{mg} / \mathrm{L}$; a su vez se determinó un caudal promedio de $0.82 \mathrm{l} / \mathrm{s}$, caudal mínimo $0.45 \mathrm{l} / \mathrm{s}$ y $1.99 \mathrm{l} / \mathrm{s}$ de caudal máximo. En base al caudal y la calidad del agua proveniente del beneficio animal, es que se determinó como necesarias las siguientes unidades: cribas, sedimentador primario, trampa de grasas, R.A.F.A., sedimentador secundario y nitrificación-nitrificación. Se planteó una propuesta para la reducción de ingreso de residuos al tratamiento de aguas residuales del beneficio animal, siendo este plasmado en una serie de recomendaciones para la valorización y correcto manejo de residuos generados en el proceso de beneficio.

De acuerdo a la observación se recomienda: Reemplazar el proceso de evisceración por el uso de pistolas de agua a presión. La sangre generada en el proceso de desollado puede ser almacenada 
con el fin de poder ser aprovechada, pudiéndose convertir en morcilla, harina de sangre y/o complemento alimenticio para porcinos. Es recomendable la sensibilización a usuarios y matarifes en evitar el arrojo de sólidos (vísceras, cachos, botellas plásticas, entre otros) a las canaletas que conducen el agua sin tratar. Se recomienda cambiar el cubrimiento del canal por rejillas de platina en su totalidad o por alguna estrategia similar. Se recomienda la implementación de una planta de tratamiento de aguas residuales donde se considere como unidades mínimas: cribas, sedimentador primario, trampa de grasas, un tratamiento secundario (ya sea aerobio o anaerobio), sedimentador secundario y un tratamiento terciario de nitrificación-desnitrificación. Dar mantenimiento periódico a las unidades existentes, retirando los lodos generados y residuos sólidos varios que llega a la PTAR. Se recomienda que se implemente un cronograma anual de mantenimiento ala cisterna encargada del transporte y vertimiento de las aguas residuales, permitiendo esto, prevenir posibles problemas mecánicos o de hermeticidad de la cisterna. Se recomienda la entrega de elementos de protección personal adecuados a los trabajadores encargados de la disposición final, tales como guantes, mascarillas, overoles, entre otros; a suvez, brindarles información respecto a los riegos a los que están expuestos. Se recomienda realizar una caracterización detallada del agua residual generada en planta en futuras investigaciones, a fin de determinar la diferencia de concentraciones de parámetros al momento de beneficio y limpieza de planta, primordialmente, el grado de concentración de cloro, el cual podría afectar la cantidad de microorganismo en un tratamiento biológico. A su vez, se recomienda que en el diseño de un nuevo sistema de tratamiento de aguas residuales se considere el crecimiento de la población y por ende del consumo de carne con el fin de evitar un colapso del tratamiento a futuro,siendo este diseñado por un especialista en el material tal como un ingenierosanitario. Dar cumplimiento al plan de fumigación y desratización. Mantener la estrategia para mitigar olores del tratamiento de aguas residualesen base a cal. Adicionar las buenas prácticas hacia el medio ambiente en el reglamento interno bajo el que se rige el matadero municipal.

\section{Referencias Bibliográficas}

Benavides Benavides, L. d. (2006). Evaluación de la planta de tratamientos de aguasresiduales de la central de sacrifivio de Túquerres (Nariño). Manizales.

Bustillo-Lecompte, C. F. (15 de septiembre de 2015). Slaughterhouse wastewater characteristics, treatment, andmanagement in the meat processing industry: A review on trends and advances. Journal of Environmental Management, 161, 287-302. Recuperado el 14 de octubre de 2017, de http://www.sciencedirect.com.bdatos.usantotomas.edu.co:2048/science/articl e/pii/S0301479715301535

Cammarota, M., (2006). A review on hydrolytic enzymes in the treatment of wastewater with high oil and grease content. Bioresource Technology, 97(17),2195-2210.

Espinoza Peralta, S. (2017). Alternativas de tratamiento de agua residuales del camalmunicipal del Distrito de Tumán. Chiclayo.

Gilberto Salas C., C. C. (2008). Tratamiento de las aguas residuales deun centro de beneficio o matadero de ganado. Revista Peruana

de Química e Ingeniería Química, XI(1), 29-35. Recuperado el 16 de octubrede 2017

Instituto Nacional de Estadística e Informática. (2010). Clasificacion Industrial Internacional Uniforme. Lima.

Instituto Nacional de Estadística e Informática. (2018). Resultados Definitivos de losCensos Nacionales 2017-Tacna (Vol. $\quad$ I). Obtenido de https://www.inei.gob.pe/media/MenuRecursivo/publicaciones_digitales/Est/Lib1564/

Ministerio de Agricultura y Riego. (2015). Manual $N^{\circ} 5$ Medición de agua. Lima. 
Mittal, G. (2006). Treatment of wastewater from abattoirs before land application.

Bioresource Technology, 97(9), 1119-1135.

Municipalidad Provincial de Tacna. (2015). Mantenimiento del camalmunicipal "MarioEyzaguirre Yañez" de Tacna. Plan de Trabajo, Municipalidad Provincial de Tacna, Tacna.

OEFA. (2017). Organismo de Evaluación y Fiscalización Ambiental. Recuperado el Julio de 2019, de https://www.oefa.gob.pe/?wpfb_dl=7827

Taveras, M. A., Silva, M., Flores Chang, F., \& de León, M. (2011). Guía para BuenasPrácticas Ambientales en Mercados y Mataderos. República Dominicana. 